\title{
Confirmation of the Sequence of 'Candidatus Liberibacter asiaticus' and Assessment of Microbial Diversity in Huanglongbing-Infected Citrus Phloem Using a Metagenomic Approach
}

\author{
Heather L. Tyler, ${ }^{1}$ Luiz F. W. Roesch, ${ }^{1}$ Siddarame Gowda, ${ }^{2}$ William O. Dawson, ${ }^{2}$ and Eric W. Triplett ${ }^{1}$ \\ ${ }^{1}$ Department of Microbiology and Cell Science, University of Florida, P.O. Box 110700, Gainesville 32611-0700, \\ U.S.A.; ${ }^{2}$ Department of Plant Pathology, University of Florida, Citrus Research and Education Center, 700 Experiment \\ Station Road, Lake Alfred 33850, U.S.A.
}

Submitted 9 June 2009. Accepted 9 July 2009.

\begin{abstract}
The citrus disease Huanglongbing (HLB) is highly destructive in many citrus-growing regions of the world. The putative causal agent of this disease, 'Candidatus Liberibacter asiaticus', is difficult to culture, and Koch's postulates have not yet been fulfilled. As a result, efforts have focused on obtaining the genome sequence of ' $\mathrm{Ca}$. $\mathrm{L}$. asiaticus' in order to give insight on the physiology of this organism. In this work, three next-generation high-throughput sequencing platforms, 454, Solexa, and SOLiD, were used to obtain metagenomic DNA sequences from phloem tissue of Florida citrus trees infected with HLB. A culture-independent, polymerase chain reaction (PCR)-independent analysis of $16 S$ ribosomal RNA sequences showed that the only bacterium present within the phloem metagenome was ' $\mathrm{Ca} \mathrm{L}$. asiaticus'. No viral or viroid sequences were identified within the metagenome. By reference assembly, the phloem metagenome contained sequences that provided 26-fold coverage of the ' $\mathrm{Ca}$. L. asiaticus' contigs in GenBank. By the same approach, phloem metagenomic data yielded less than 0.2-fold coverage of five other alphaproteobacterial genomes. Thus, phloem metagenomic DNA provided a PCR-independent means of verifying the presence of ' $\mathrm{Ca}$ L. asiaticus' in infected tissue and strongly suggests that no other disease agent was present in phloem. Analysis of these metagenomic data suggest that this approach has a detection limit of one ' $\mathrm{Ca}$. Liberibacter' cell for every 52 phloem cells. The phloem sample sequenced here is estimated to have contained 1.7 ' $\mathrm{Ca}$. Liberibacter' cells per phloem cell.
\end{abstract}

Huanglongbing (HLB), also known as citrus greening, is a destructive and devastating disease of citrus causing great losses in citrus industries throughout the world (Bove 2006). This disease is characterized by yellow shoots, blotchy mottle

All metagenomic sequences are available in the National Center for Biotechnolgoy Information (NCBI) Short Read Archive under accession numbers SRR016809, SRR016816, and SRR017902. Accession numbers for 454 and SOLiD data are SRR016809 and SRR017902. ARISA band sequences are available in the GenBank database under accession numbers GQ254546 to GQ254633.

Corresponding author: E. W. Triplett; E-mail address: ewt@ufl.edu

* The $e$-Xtra logo stands for "electronic extra" and indicates six supplemental tables are published online and that Figure 1 appears in color online. on the leaves, and fruit that are small and lop-sided, with inverted coloring. HLB is transmitted between citrus trees by the psyllids Diaphorina citri (in Asia and America) and Trioza erytreae (in Africa) (McClean and Oberholzer 1965; Capoor et al. 1967; Bove 2006). Progress in the study of this disease, including detection, treatment, and control, has been hindered due, in part, to the lack of effective culturing. Through microscopic examination and sequence analysis of polymerase chain reaction (PCR)-amplified 16S ribosomal DNA of infected tissue, it has been proposed that the causal agent of citrus greening is a phloem sieve tube-limited gram-negative bacterium that is a member of the alphaproteobacteria (Garnier et al. 1984; Jagoueix et al. 1994). More recent studies have found HLB to be associated with three members of the genus 'Candidatus Liberibacter': ' $C a$. Liberibacter asiaticus', ' $C a$. L. americanus', and ' $\mathrm{Ca}$. L. africanus' (Jagoueix et al. 1997; Teixeira et al. 2005).

In order to get around the obstacle of ' $\mathrm{Ca}$. Liberibacter spp.' being uncultured, attempts have been made to sequence the genome directly from infected tissue, in the hopes that knowledge of the genome will give better insight into how to culture this organism as well as how to combat it. Although ' $\mathrm{Ca}$. L. asiaticus' is at a low titer in citrus trees, it is present in much higher numbers when infected in periwinkle and Dodder (Ghosh et al. 1978; Garnier and Bove 1983). Therefore, DNA from infected plants in this system has been sequenced to obtain 8.56- and 14.7-kb segments of the ' $\mathrm{Ca}$. Liberibacter' genome using a genomic walking method (Doddapaneni et al. 2008; Lin et al. 2008). Recently, 34 contigs of ' $\mathrm{Ca}$ L. asiaticus' str. psy62 genomic sequence, ranging in size from 1.033 to $186.24 \mathrm{~kb}$ for a total of $1.2 \mathrm{Mb}$ of genomic sequence, has been deposited into GenBank (GenBank accession number ABQW00000000). These genomic sequences were obtained from bacteria extracted from the psyllid vector (Duan et al. 2009). At the time of our study, only 34 contigs were available for analysis, although the genome has been closed and is now published (Duan et al. 2009).

Therefore, in this article, we set out to confirm that the proposed ' $\mathrm{Ca}$. L. asiaticus' contigs are, indeed, associated with HLB-symptomatic plants and not asymptomatic plants. Most HLB-infected trees in Florida are also infected with Citrus tristeza virus (CTV). We examined a CTV-free HLB isolate that was used to characterize the host range and symptoms of Florida HLB (Folimonova et al. in press). In addition, a nonPCR-based metagenomic approach was used to investigate the 
microbial diversity within HLB-infected citrus tissue and whether there are any other pathogens present.

\section{RESULTS}

Verification of ' $C a$. L. asiaticus' str. psy62 contigs.

In order to confirm that the ' $\mathrm{Ca}$. L. asiaticus' contigs deposited in GenBank belong to the causative agent of HLB, it was necessary to verify that these contigs are present only in infected citrus tissue and not in healthy tissue. To that end, PCR reactions to assay for the presence of each contig were performed with genomic DNA from healthy and infected Citrus sinensis tissue. Before PCR, the 34 putative ' $\mathrm{Ca}$. L. asiaticus' contigs in GenBank were assembled and reduced to 32, with NZ_ABQW01000005 assembling with NZ_ABQW01000033 and NZ_ABQW01000022 assembling with NZ_ABQW01000031. Of these 32 contigs, 30 were found to have bands of the expected size range in infected tissue but not healthy tissue (data not shown), confirming that these sequences are, indeed, associated with HLB symptoms and, therefore, from the likely causative agent of the disease. Of the two contigs that were not

Table 1. Overview of metagenomic sequence statistics

\begin{tabular}{lccc}
\hline $\begin{array}{l}\text { Sequencing } \\
\text { technique }\end{array}$ & $\begin{array}{c}\text { No. of } \\
\text { reads }\end{array}$ & $\begin{array}{c}\text { No. of } \\
\text { gigabases }\end{array}$ & $\begin{array}{c}\text { Average read } \\
\text { length }\end{array}$ \\
\hline 454 & 246,355 & 0.058 & 238.48 \\
Solexa & $49,423,731$ & 1.8 & 36 \\
SOLiD & $337,075,448$ & 11.8 & 35 \\
All Data & $386,745,534$ & 13.6 & 35.26 \\
\hline
\end{tabular}

confirmed, there were either positive bands in both infected and healthy reactions or no positive bands in either reaction. These results could be explained by nonspecific primer binding to regions in the $C$. sinensis genome.

\section{Confirmation of ' $\mathrm{Ca}$. L. asiaticus' contig sequences.}

Because ' $\mathrm{Ca}$. L. asiaticus' contigs were detected only in infected tissue by PCR, a metagenomics approach was used to confirm that the entire sequence of each contig was present in HLB-infected phloem, further verifying that the sequences are from the causative agent of the disease. To do this, three nextgeneration sequencing platforms, 454, SOLiD, and Solexa, were used to generate a total of 13.6 gigabases of metagenomic sequencing reads from HLB-infected citrus phloem (Table 1). With the large amount of sequence data obtained from HLB-infected phloem, high coverage of the ' $\mathrm{Ca}$ L. asiaticus' genome and, therefore, the ' $\mathrm{Ca}$. L. asiaticus' contigs, was expected. To determine the level of ' $\mathrm{Ca}$. L. asiaticus' coverage, all metagenomic sequencing data were run in a reference assembly against the ' $\mathrm{Ca}$ L. asiaticus' contigs. Of all phloem sequences, $0.23 \%$ matched the ' $C a$. L. asiaticus' contigs, with coverage ranging from 20 - to 60 -fold per contig (Table 2). In addition, sequences from HLB-infected phloem spanned the entire length of each contig, with 99.69 to $100 \%$ of each contig matching the consensus sequence generated from the reference assembly (Table 3 ).

The dataset from each high-throughput sequencing method was also run against the ' $\mathrm{Ca}$ L. asiaticus' contigs separately in order to determine whether there were any biases due to sequencing method. One of the major differences between the

Table 2. Fold coverage of each 'Candidatus Liberibacter asiaticus' contig determined by reference assembly with 454, Solexa, and SOLiD metagenomic data from Huanglongbing-infected citrus phloem ${ }^{\mathrm{a}}$

\begin{tabular}{|c|c|c|c|c|}
\hline \multirow[b]{2}{*}{ Reference sequences } & \multicolumn{4}{|c|}{ Fold coverage } \\
\hline & 454 & Solexa & SOLiD & All data sets \\
\hline NZ_ABQW01000001 & 0.054 & 14.314 & 12.221 & 26.595 \\
\hline NZ_ABQW01000002 & 0.056 & 15.028 & 12.005 & 27.089 \\
\hline NZ_ABQW01000003 & 0.08 & 13.949 & 9.146 & 23.175 \\
\hline NZ_ABQW01000004 & 0.083 & 15.048 & 13.107 & 28.272 \\
\hline NZ_ABQW01000005_33 & 0.057 & 15.414 & 11.843 & 27.314 \\
\hline NZ_ABQW01000006 & 0.081 & 14.459 & 10.881 & 25.421 \\
\hline NZ_ABQW01000007 & 0.061 & 16.453 & 12.288 & 28.802 \\
\hline NZ_ABQW01000008 & 0.074 & 13.405 & 9.257 & 22.736 \\
\hline NZ_ABQW01000009 & 0.101 & 13.624 & 11.64 & 25.348 \\
\hline NZ_ABQW01000010 & 0.026 & 13.835 & 8.996 & 22.855 \\
\hline NZ_ABQW01000011 & 0.055 & 12.495 & 15.286 & 27.789 \\
\hline NZ_ABQW01000012 & 0.033 & 13.196 & 9.464 & 22.694 \\
\hline NZ_ABQW01000013 & 0.046 & 14.242 & 11.206 & 25.494 \\
\hline NZ_ABQW01000014 & 0.083 & 13.883 & 9.675 & 23.641 \\
\hline NZ_ABQW01000015 & 0.049 & 14.103 & 8.957 & 23.109 \\
\hline NZ_ABQW01000016 & 0.07 & 19.019 & 9.921 & 29.009 \\
\hline NZ_ABQW01000017 & 0.064 & 14.895 & 10.927 & 25.886 \\
\hline NZ_ABQW01000018 & 0.112 & 10.782 & 16.71 & 27.599 \\
\hline NZ_ABQW01000019 & 0.124 & 14.705 & 9.243 & 24.071 \\
\hline NZ_ABQW01000020 & 0.197 & 14.564 & 15.644 & 30.404 \\
\hline NZ_ABQW01000021 & 0.055 & 13.022 & 11.739 & 24.816 \\
\hline NZ_ABQW01000022_31 ${ }^{\mathrm{c}}$ & 0.341 & 22.494 & 29.517 & 52.363 \\
\hline NZ_ABQW01000023 & 0.164 & 16.9 & 20.3 & 37.364 \\
\hline NZ_ABQW01000024 & 0.088 & 11.734 & 13.978 & 25.8 \\
\hline NZ_ABQW01000025 & 0.099 & 26.224 & 14.892 & 41.211 \\
\hline NZ_ABQW01000026 & 0.219 & 10.862 & 18.077 & 28.904 \\
\hline NZ_ABQW01000027 & 0.106 & 15.764 & 17.352 & 33.209 \\
\hline NZ_ABQW01000028 & 0 & 15.026 & 5.616 & 20.642 \\
\hline NZ_ABQW01000029 & 0 & 14.958 & 11.047 & 26.042 \\
\hline NZ_ABQW01000030 & 0 & 13.966 & 8.51 & 22.477 \\
\hline NZ_ABQW01000032 & 0.333 & 28.444 & 31.289 & 60.067 \\
\hline NZ_ABQW01000034 & 0 & 15.95 & 12.042 & 27.913 \\
\hline
\end{tabular}

${ }^{\mathrm{a}}$ Each contig represented by its GenBank Ref Seq number.

${ }^{\mathrm{b}}$ Contig sequence assembled from NZ_ABQW01000005 and NZ_ABQW01000033 using SeqMan Pro.

${ }^{\mathrm{c}}$ Contig sequence assembled from NZ_ABQW01000022 and NZ_ABQW01000031 using SeqMan Pro. 
results of the three sequencing methods appears to be due to number of reads, with the $58.8 \mathrm{Mb}$ of 454 sequences only yielding 0 - to 0.341 -fold coverage of the ' $C a$. L. asiaticus' contigs (Table 2). In contrast, reference assemblies with Solexa and SOLiD data, which provide significantly more sequences (1.78 and $11.8 \mathrm{~Gb}$ ), yielded 10.78 - to 28.44 -fold and 5.62- to 31.29-fold coverage, respectively, of the ' $\mathrm{Ca}$. L. asiaticus' contigs (Table 2 ). When looking at the consensus sequences generated by these two reference assemblies, Solexa sequences covered 98.9 to $100 \%$ of each ' $\mathrm{Ca}$ L. asiaticus' contig and SOLiD data covered 82.39 to $100 \%$ (Table 3). Of the three sequencing methods, Solexa yielded slightly better fold coverage of the ' $C a$. L. asiaticus' contigs overall. For example, 23 of the 32 ' $C a$. Liberibacter spp.' contigs had higher fold coverage when assembled against Solexa data than with 454 or SOLiD (Table 2). Specifically, contig NZ_ABQW01000028 had 5.62fold coverage with SOLiD sequences but, when assembled with Solexa sequences, fold coverage of this contig almost tripled, going up to 15.03 (Table 2). There were also cases where SOLiD data yielded higher fold coverage of a contig but the increase was not as much. In one such case, contig NZ_ABQW01000026 had 18.077-fold coverage with SOLiD data and 10.862 with Solexa (Table 2). Sequences from 454 did not approach the level of coverage seen using SOLiD or Solexa for any of the contigs, mostly due to the reduced number of sequences from 454 compared with the other two. For example, 454 yielded 0.25 million reads whereas SOLiD and Solexa yielded 337 and 49 million reads, respectively. However, overall, sequences from the three methods complemented gaps missed by the other two because, when analyzed sepa- rately, only 4 of the 32 ' $\mathrm{Ca}$. L. asiaticus' contigs had consensus sequences that covered $100 \%$ of their length but, when all data were assembled together against the ' $\mathrm{Ca}$. L. asiaticus' contigs, 11 of those contigs had a $100 \%$ match (Table 3 ). With such high fold coverage and consensus sequences covering the entirety of each contig, it was concluded that these contigs are present in the phloem.

\section{Comparison to alphaproteobacterial relatives.}

In order to discount the possibility that the matches seen when comparing citrus phloem sequences to ' $\mathrm{Ca}$. L. asiaticus' contigs are due to the presence of close alphaproteobacterial relatives, all infected phloem sequence data was compared with five fully sequenced alphaproteobacteria deemed to be closely related to ' $\mathrm{Ca}$. L. asiaticus' after a BLAST of its contigs against an alphaproteobacterial database. The results of the reference assemblies with each of the five alphaproteobacteria showed that over 100-fold fewer sequencing reads match these alphaproteobacteria compared with ' $\mathrm{Ca}$. L. asiaticus' contigs (Table 4). For example, the closest match, Rhizobium leguminosarum bv. viciae 3841, had 8,012 reads match its genome while 900,124 reads matched the ' $C a$. L. asiaticus' contigs. In addition, sequencing reads from infected phloem yielded an average 26.34-fold coverage of the ' $\mathrm{Ca}$ L. asiaticus' contigs but none of the alphaproteobacteria had greater than 0.175 -fold coverage (Table 4). Therefore, the bacterial DNA in phloem is specific to ' $\mathrm{Ca}$ L. asiaticus' and even close relatives do not match this DNA. This confirmation of ' $\mathrm{Ca}$ L. asiaticus' in symptomatic plant tissue further supports its proposed role as the causative agent of HLB.

Table 3. Percent length of each 'Candidatus Liberibacter asiaticus' contig covered by 454, Solexa, and SOLiD metagenomic data from Huanglongbinginfected citrus phloem as determined by reference assembly ${ }^{\mathrm{a}}$

\begin{tabular}{|c|c|c|c|c|}
\hline \multirow[b]{2}{*}{ Reference sequences } & \multicolumn{4}{|c|}{ Contig length covered by phloem sequences (\%) } \\
\hline & 454 & Solexa & SOLiD & All data sets \\
\hline NZ_ABQW01000001 & 4.951 & 99.947 & 94.585 & 99.989 \\
\hline NZ_ABQW01000002 & 4.966 & 99.973 & 94.272 & 99.994 \\
\hline NZ_ABQW01000003 & 6.826 & 99.930 & 91.246 & 99.966 \\
\hline NZ_ABQW01000004 & 7.413 & 99.671 & 93.607 & 99.784 \\
\hline NZ_ABQW01000005_33 b & 5.022 & 99.936 & 92.817 & 99.947 \\
\hline NZ_ABQW01000006 & 7.205 & 99.991 & 92.966 & 99.999 \\
\hline NZ_ABQW01000007 & 4.917 & 99.927 & 92.189 & 99.946 \\
\hline NZ_ABQW01000008 & 6.635 & 99.970 & 91.620 & 99.995 \\
\hline NZ_ABQW01000009 & 7.100 & 99.891 & 93.702 & 99.997 \\
\hline NZ_ABQW01000010 & 2.545 & 99.928 & 91.094 & 99.964 \\
\hline NZ_ABQW01000011 & 3.075 & 99.393 & 95.527 & 99.691 \\
\hline NZ_ABQW01000012 & 3.295 & 99.927 & 93.125 & 100.002 \\
\hline NZ_ABQW01000013 & 3.899 & 99.964 & 93.291 & 99.964 \\
\hline NZ_ABQW01000014 & 3.223 & 99.952 & 91.694 & 99.952 \\
\hline NZ_ABQW01000015 & 4.942 & 99.964 & 91.593 & 100.000 \\
\hline NZ_ABQW01000016 & 6.984 & 99.991 & 91.402 & 99.995 \\
\hline NZ_ABQW01000017 & 5.456 & 99.989 & 93.582 & 99.989 \\
\hline NZ_ABQW01000018 & 7.112 & 99.469 & 98.340 & 100.000 \\
\hline NZ_ABQW01000019 & 10.086 & 99.983 & 93.184 & 99.983 \\
\hline NZ_ABQW01000020 & 16.012 & 99.939 & 97.128 & 99.983 \\
\hline NZ_ABQW01000021 & 4.232 & 99.893 & 94.603 & 100.000 \\
\hline NZ_ABQW01000022_31 $1^{\mathrm{c}}$ & 28.651 & 99.525 & 97.375 & 99.835 \\
\hline NZ_ABQW01000023 & 14.740 & 99.880 & 97.985 & 100.000 \\
\hline NZ_ABQW01000024 & 4.783 & 100.000 & 97.285 & 100.000 \\
\hline NZ_ABQW01000025 & 9.843 & 99.837 & 91.628 & 99.837 \\
\hline NZ_ABQW01000026 & 21.144 & 98.900 & 95.869 & 99.951 \\
\hline NZ_ABQW01000027 & 10.614 & 100.000 & 99.321 & 100.000 \\
\hline NZ_ABQW01000028 & 0.000 & 99.854 & 82.389 & 99.854 \\
\hline NZ_ABQW01000029 & 0.000 & 99.849 & 96.939 & 100.000 \\
\hline NZ_ABQW01000030 & 0.000 & 99.873 & 90.361 & 100.000 \\
\hline NZ_ABQW01000032 & 25.874 & 99.563 & 100.000 & 100.000 \\
\hline NZ_ABQW01000034 & 0.000 & 100.000 & 96.902 & 100.000 \\
\hline
\end{tabular}

${ }^{\mathrm{a}}$ Each contig represented by its GenBank Ref Seq number.

${ }^{\mathrm{b}}$ Contig sequence assembled from NZ_ABQW01000005 and NZ_ABQW01000033 using SeqMan Pro.

${ }^{\mathrm{c}}$ Contig sequence assembled from NZ_ABQW01000022 and NZ_ABQW01000031 using SeqMan Pro. 


\section{Assessment of bacterial diversity.}

Automated ribosomal intergenic spacer analysis (ARISA) was performed for the initial assessment of bacterial diversity within HLB-infected phloem. Amplification with ARISA primers yielded two dominant peaks in healthy (740 and 1,330 bp) and three dominant peaks in infected (740, 1,330, and 1,600 bp) phloem (Fig. 1). To determine the identity of these peaks, the ARISA PCR was run on a gel and the bands extracted, cloned, and sequenced in a 96-well plate. The 740- and 1,330-bp bands were from $C$. sinensis chloroplast and mito- chondria, respectively, while the 1,600-bp band unique to infected tissue was from ' $\mathrm{Ca}$. Liberibacter' $16 \mathrm{~S}$ ribosomal (r)RNA. Of all the sequences obtained from the 1,600-bp band, there was a 100-fold excess of ' $\mathrm{Ca}$. Liberibacter' sequences, indicating it as the most abundant bacterium present within the phloem.

Once the ' $\mathrm{Ca}$ L. asiaticus' contigs were confirmed in infected citrus tissue, metagenomic sequencing data from the infected phloem was analyzed to further assess whether ' $\mathrm{Ca} \mathrm{L}$. asiaticus' was the only bacterium present in this sample. Bac-

Table 4. All metagenomic phloem sequences compared with the contigs of 'Candidatus Liberibacter asiaticus' or the fully sequenced genomes of other alphaproteobacteria by reference assembly

\begin{tabular}{|c|c|c|c|c|}
\hline Bacterial species & Genome size (bp) & No. of matching reads & No. of matching bases & Fold coverage \\
\hline 'Ca. L. asiaticus' str. psy62 & $1,217,424$ & 900,124 & $32,071,418$ & 26.34 \\
\hline Rhizobium leguminosarum bv. viciae 3841 & $5,892,908$ & 8,027 & 281,989 & 0.048 \\
\hline Bartonella bacilliformis KC583 & $1,445,021$ & 7,209 & 253,180 & 0.175 \\
\hline Caulobacter crescentus CB15 & $4,016,947$ & 5,969 & 209,512 & 0.052 \\
\hline Brucella abortus S19 & $2,238,636$ & 7,505 & 263,576 & 0.118 \\
\hline Orientia tsutsugamushi str. Boryong & $2,127,051$ & 2,339 & 82,333 & 0.039 \\
\hline
\end{tabular}
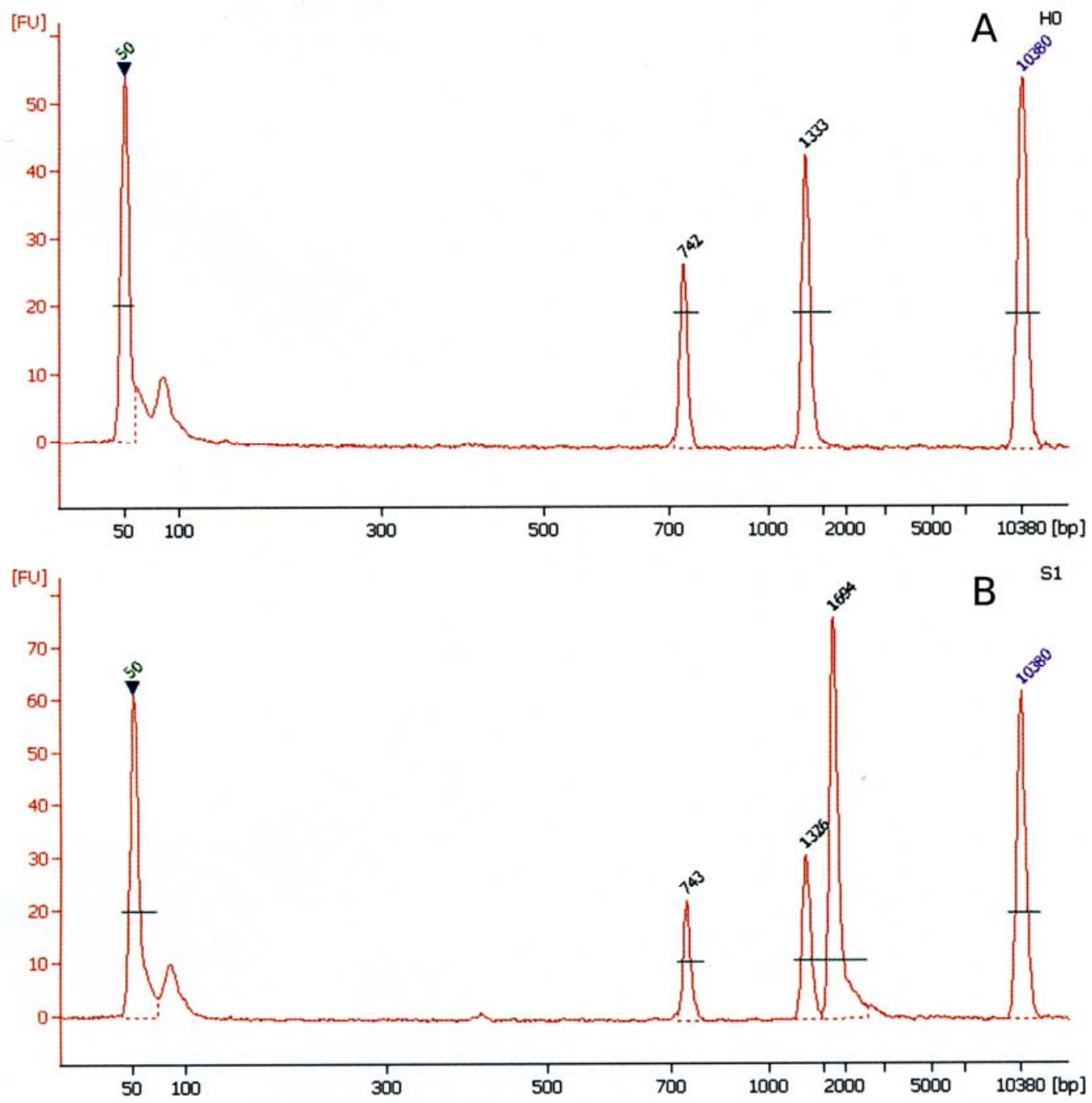

Fig. 1. Automated ribosomal intergenic spacer analysis (ARISA) of healthy and Huanglongbing-infected Citrus sinensis phloem. Example of the ARISA profiles for the DNA isolated from the phloem tissue of citrus samples (A, nonsymptomatic and B, symptomatic) generated by the Agilent 2100 bioanalyzer. Base pair sizes are indicated adjacent to the peaks. The left-most $(50 \mathrm{bp})$ and the right-most $(10,380 \mathrm{bp})$ peaks represent the markers used to align the ladder data with data from the sample wells. The peaks of approximately $740 \mathrm{bp}$ represent sequences amplified from the $C$. sinensis chloroplast, the peaks of approximately $1,330 \mathrm{bp}$ represent the sequences amplified from the mitochondrial gene for mitochondrial RNA, and the peak of approximately $1,600 \mathrm{bp}$ corresponds of the sequences amplified from 'Candidatus Liberibacter asiaticus'. 
terial diversity in the phloem of $C$. sinensis plants infected with ' $C a$ L. asiaticus' was analyzed by searching total DNA sequences obtained from infected phloem for 16S rRNA gene sequences. Because plant chloroplasts are related to Cyanobacteria, plant chloroplast sequences were filtered out of the phloem sequences. To do this, the entire dataset was assembled against the $C$. sinensis chloroplast genome and nonassembled reads were taken for further analysis. Approximately 5.7 million of the total sequencing reads matched the chloroplast genome and were removed. The remaining 381,056,442 sequences were run in a reference assembly against the Ribosomal Database Project (RDP) database. Sequences from the 454, Solexa, and SOLiD data sets were run both separately and together. Each reference assembly yielded an output with the number of sequencing reads matching each reference in the database, average coverage of each $16 \mathrm{~S}$ rRNA match, and length of the consensus sequence matching each 16S rRNA gene. In each assembly, the $16 \mathrm{~S}$ rRNA sequence from the database with the greatest number of matches to infected citrus phloem sequence was ' $\mathrm{Ca}$. Liberibacter' (Supplementary Tables $\mathrm{S} 1, \mathrm{~S} 2, \mathrm{~S} 3$, and S4). In addition, the ' $\mathrm{Ca}$. Liberibacter' $16 \mathrm{~S}$ rRNA gene had the highest fold coverage and longest consensus sequence over the entirety of the gene compared with any other bacteria in the database (Table 5). In total, the phloem metagenome from all three sequencing platforms combined yielded a fold coverage of 23.17 of the ' $\mathrm{Ca}$. Liberibacter' $16 \mathrm{~S}$ rRNA gene, with a consensus sequence of 1,212 bp in length (Table 6). The length of the ' $C a$. Liberibacter' 16S rRNA sequence in the database was $1,448 \mathrm{bp}$; therefore, $83.7 \%$ of the 16S rRNA gene was covered (Table 6). In contrast, the next highest match, the 16S rRNA gene from Nitrospina gracilis, had a fold coverage of 1.45 and a consensus sequence that only covered $7.15 \%$ of the gene. All other matches to members of the RDP database covered less than $14 \%$ of the total $16 \mathrm{~S}$ rRNA gene. Even coverage over the entire length of the $16 \mathrm{~S}$ rRNA gene is expected for any bacterium present in the phloem; therefore, ' $\mathrm{Ca}$. Liberibacter' is likely the only bacterium present. In addition, the short regions within the $16 \mathrm{~S}$ gene of the other RDP matches had 90 to $100 \%$ identity with sequences in ' $\mathrm{Ca}$ L. asiaticus' and citrus chloroplast and mitochondria when compared against these references on GenBank. Therefore, it was concluded that these matches were false positives. Given that it would be impossible eliminate all falsepositive matches due to alignment and sequencing errors, a threshold fold coverage detection limit was determined. In order to calculate this detection limit, the mean and standard deviation were calculated from the fold coverage of every RDP 16S rRNA match. Any RDP match with a fold coverage greater than the mean plus 3 standard deviations (1.89) was deemed to be significant and above the threshold fold coverage that would be seen with any $16 \mathrm{~S}$ rRNA gene assembling to remaining citrus and ' $\mathrm{Ca}$. Liberibacter' sequences due to misalignments or sequencing errors. Because ' $\mathrm{Ca}$. Liberibacter' was the only RDP database member to have coverage above 1.89 , it was deemed to be the only bacterium likely present in citrus phloem.

Similar results were seen when SOLiD and Solexa data were run against the RDP database separately, yielding a fold coverage of the ' $C a$. Liberibacter' $16 \mathrm{~S}$ rRNA of 17.37 and 5.23 , respectively (Table 6). When 454 sequences were run separately, fold coverage was 0.33 , with a consensus sequence matching only $26.17 \%$ of the ' $\mathrm{Ca}$. Liberibacter' $16 \mathrm{~S}$ rRNA gene (Table 6). This number is not entirely surprising because very few 454 reads matched any of the $16 \mathrm{~S}$ rRNA sequences. Given that the average read length of the 454 sequences was $248 \mathrm{bp}$ and only two of those reads matched the 1,448-bp ' $\mathrm{Ca}$.

Table 5. Fold coverage of the top $16 \mathrm{~S}$ ribosomal RNA Ribosomal Database Project database matches with 454, Solexa, and SOLiD metagenomic data from Huanglongbing-infected citrus phloem

\begin{tabular}{|c|c|c|c|c|}
\hline \multirow[b]{2}{*}{ Reference sequences } & \multicolumn{4}{|c|}{ Fold coverage } \\
\hline & 454 & Solexa & SOLiD & All data sets \\
\hline 'Candidatus Liberibacter spp.' & 0.328 & 5.23 & 17.365 & 23.173 \\
\hline Nitrospina gracilis & 0 & 0 & 1.427 & 1.451 \\
\hline 'Candidatus Burkholderia spp.' & 0 & 0.375 & 0.885 & 1.301 \\
\hline Syntrophus gentianae & 0 & 0.046 & 0.603 & 1.028 \\
\hline Nitratifractor salsuginis & 0 & 0 & 0.942 & 0.991 \\
\hline S. buswellii & 0 & 0.022 & 0.51 & 0.895 \\
\hline Pseudomonas rhizosphaerae & 0 & 0.023 & 0.616 & 0.886 \\
\hline Hoeflea marina & 0 & 0.073 & 1.229 & 0.85 \\
\hline Thermonema rossianum & 0 & 0 & 0.766 & 0.837 \\
\hline Thermodesulfovibrio islandicus & 0 & 0.231 & 0.645 & 0.804 \\
\hline Leucothrix mucor & 0 & 0.094 & 0.685 & 0.802 \\
\hline Desulfobacter halotolerans & 0 & 0.073 & 0.448 & 0.732 \\
\hline$P$. argentinensis & 0 & 0 & 1.049 & 0.685 \\
\hline Desulfospira joergensenii & 0 & 0.048 & 0.506 & 0.627 \\
\hline Martelella mediterranea & 0 & 0 & 0.993 & 0.615 \\
\hline Marinomonas aquimarina & 0 & 0 & 0.509 & 0.554 \\
\hline Mycoplasma cloacale & 0 & 0.025 & 0.427 & 0.527 \\
\hline Phaeospirillum fulvum & 0 & 0.051 & 0.448 & 0.525 \\
\hline Desulfotignum phosphitoxidans & 0 & 0.074 & 0.508 & 0.508 \\
\hline Desulfomonile tiedjei & 0 & 0.14 & 0.369 & 0.508 \\
\hline Desulfobacula toluolica & 0.03 & 0 & 0.223 & 0.345 \\
\hline
\end{tabular}

Table 6. Comparison of 'Candidatus Liberibacter asiaticus' 16S ribosomal RNA to 454, Solexa, and SOLiD metagenomic sequences from Huanglongbinginfected phloem by reference assembly

\begin{tabular}{|c|c|c|c|c|}
\hline Sequence & No. of matching reads & Fold coverage & Length of consensus (bp) & $16 \mathrm{~S}$ gene matching consensus sequence $(\%)$ \\
\hline 454 & 2 & 0.328 & 379 & 26.17 \\
\hline Solexa & 211 & 5.23 & 950 & 65.61 \\
\hline SOLiD & 720 & 17.37 & 1,118 & 77.21 \\
\hline All sequences & 944 & 23.17 & 1,212 & 83.70 \\
\hline
\end{tabular}


Liberibacter' $16 \mathrm{~S}$ rRNA gene, a match to only $26 \%$ of the $16 \mathrm{~S}$ rRNA is unsurprising. There was only one other species in the entire database that had a matching member when run against the 454 data and it had 100-fold lower coverage than ' $\mathrm{Ca} \mathrm{L}$. asiaticus' (Table 5). As a result, the 454 data had little effect on the results of citrus phloem community analysis using the RDP database. It is likely this is due to the low amount of sequence generated by 454 compared with the other two methods. It is clear, however, that both SOLiD and Solexa contributed to the community analysis, because the overall fold coverage determined by the reference assembly using all three sets of sequencing data was higher than the fold coverage found when analyzing these datasets separately (Table 6).

\section{Other reported HLB-associated bacteria not present.}

In addition to ' $\mathrm{Ca}$. Liberibacter spp.', other bacteria have been reported to be associated with HLB infection in citrus, including Propionibacterium acnes, ' $\mathrm{Ca}$. Phytoplasma asteri', and pigeon pea witches'-broom phytoplasma (Davis et al. 2008; Teixeira et al. 2008; Chen et al. 2009). These reports are in contradiction to the results of the $16 \mathrm{~S}$ rRNA analysis of the citrus metagenome, which yielded a fold coverage of 0.023 and 0.026 for ' $C a$. P. asteri' and $P$. acnes, respectively, which corresponds to one 35-bp sequencing read matching the $16 \mathrm{~S}$ rRNA gene. To further confirm the presence or absence of these bacteria in the current study, reference assemblies of the metagenomic sequences were run against the genomes of these bacteria available on GenBank. In both cases, the fold coverage of these genomes was more than 100-fold lower than the coverage of ' $C a$. L. asiaticus' (Table 7). These results indicate that ' $C a$. P. asteri' and P. acnes are not present in the sample; otherwise, much higher coverage of these genomes would be expected if these bacteria were present in citrus phloem. In comparison, even the non-HLB-associated actinobacterial relative of $P$. acnes, Micrococcus luteus, had 0.038-fold coverage when assembled with the citrus metagenomic sequences, double the fold coverage of $P$. acnes (Table 7). Seeing a non-HLBassociated bacterium with a higher fold coverage than a bacterium reportedly associated with HLB indicates that the genome of any bacteria will match such a large amount of metagenomic sequences due to alignment with conserved genes. Upon closer inspection, 84 to $99 \%$ of the sequencing reads that matched the genomes of these three bacteria aligned to regions in the $16 \mathrm{~S}$ and $23 \mathrm{~S}$ rRNA genes. If $P$. acnes was truly present in the sample, a more even distribution of reads aligning over the genome would be expected. It is likely that a large number of these sequences matching the $16 \mathrm{~S}$ and $23 \mathrm{~S}$ rRNA genes are derived from the rRNA genes of citrus chloroplast and mitochondria, which would be present in citrus tissue in higher numbers and, therefore, inflate the fold coverage of any bacterial genome compared with the metagenome. To test this idea, rRNA regions in the reference assemblies with many metagenomic sequence matches were found to have greater than $90 \%$ and, in some cases, $100 \%$ identity with sequences from the $C$. sinensis chloroplast genome. Furthermore, when the reference assemblies were repeated with reads that had the $C$. sinensis chloroplast genome filtered out, the number of matching bases and fold coverage of ' $C a$. P. asteri', P. acnes, and M. luteus, as well as the genomes from five reference alphaproteobacteria, were significantly reduced (Table 8 ). In contrast, the fold coverage of ' $\mathrm{Ca}$ L. asiaticus' contigs remained above 26 even with chloroplast sequences removed. These analyses validate the observation that the majority of sequences matching these genomes were chloroplast in nature and these other bacterial species were not present in the phloem sample because any bacterial genome is likely to have a low level of fold coverage when compared against such a large number of metagenomic sequences due to the conserved regions in rRNA genes.

In order to test the hypothesis that ' $\mathrm{Ca}$. P. asteri' and $P$. acnes were not present in citrus phloem, a detection limit was calculated to determine the threshold level of sequencing matches required to be $99 \%$ confident that a bacterial genome is truly present in the sample. The detection limit of these metagenomic sequences was set at three standard deviations above the mean fold coverage of seven bacterial genomes referenced against the entire metagenomic dataset. At this level, any genome with a fold coverage of 0.29 or greater was considered to be above the background level of false-positive sequence matches and deemed to be present in the citrus phloem. This corresponds to $0.0021 \%$ of all bases and reads in the metagenomic dataset, or approximately 8,144 of the $386,745,534$ total reads and 285,030 bases of the total $13.6 \mathrm{~Gb}$ of metagenomic data. ' $\mathrm{Ca}$. L. asiaticus' was the only genome analyzed with a fold coverage greater than this threshold. Neither ' $C a$. P. asteri' nor $P$. acnes had enough sequencing reads match their genomes to achieve a fold coverage approaching this limit. Therefore, ' $\mathrm{Ca}$. L. asiaticus' was the only one present within these phloem samples.

Approximately $0.23 \%$ of the 13.65 billion bases sequenced in this work are from ' $\mathrm{Ca}$. Liberibacter spp.' Assuming a dip-

Table 7. Comparison of 'Candidatus Liberibacter asiaticus' and other reported Huanglongbing-associated bacteria to all metagenomic phloem sequences

\begin{tabular}{lcccc}
\hline Bacterial species & Genome size (bp) & No. of matching reads & No. of matching bases & Fold coverage \\
\hline 'Ca. L. asiaticus' str. psy62 & $1,217,424$ & 900,124 & $32,071,418$ & 26.34 \\
'Ca. Phytoplasma asteris' & 723,970 & 3,926 & 138,117 & 0.191 \\
Propionibacterium acnes KPA171202 & $2,560,265$ & 1,744 & 61,452 & 0.024 \\
Micrococcus luteus NCTC 2665 & $2,501,097$ & 2,677 & 94,125 & 0.038 \\
\hline
\end{tabular}

Table 8. Comparison of metagenomic assemblies with total metagenomic data to metagenomic data with chloroplast sequences removed

\begin{tabular}{|c|c|c|c|c|}
\hline & \multicolumn{2}{|c|}{ Fold coverage } & \multicolumn{2}{|c|}{ Bases matched } \\
\hline & Total & Minus chloroplast & Total & Minus chloroplast \\
\hline 'Candidatus Liberibacter asiaticus' str. psy62 & 26.34 & 26.179 & $32,071,418$ & $31,871,539$ \\
\hline Rhizobium leguminosarum bv. viciae 3841 & 0.048 & 0.018 & 281,989 & 104,598 \\
\hline Bartonella bacilliformis KC583 & 0.175 & 0.058 & 253,180 & 83,988 \\
\hline Caulobacter crescentus CB15 & 0.052 & 0.014 & 209,512 & 57,130 \\
\hline Brucella abortus $S 19$ & 0.118 & 0.038 & 263,576 & 84,194 \\
\hline Orientia tsutsugamushi str. Boryong & 0.039 & 0.017 & 82,333 & 35,218 \\
\hline ‘Ca. Phytoplasma asteris’ & 0.191 & 0.016 & 138,117 & 11,356 \\
\hline Propionibacterium acnes KPA171202 & 0.024 & 0.009 & 61,452 & 22,352 \\
\hline Micrococcus luteus NCTC 2665 & 0.038 & 0.004 & 94,125 & 9,822 \\
\hline
\end{tabular}


loid genome size of citrus at $900 \mathrm{Mb}$, these data represent a 15 -fold coverage of the diploid citrus genome or can be considered the full chromosomal content of 15 citrus phloem cells. Assuming a ' $\mathrm{Ca}$. Liberibacter' genome size of $1.21 \mathrm{Mb}$, the 26-fold coverage of the ' $C a$. Liberibacter' genome obtained here can be considered to be the full chromosomal content of 26 ' $\mathrm{Ca}$. L. asiaticus' cells. This suggests that the phloem sample sequenced here possessed 1.7 ' $\mathrm{Ca}$. L. asiaticus' cells per phloem cell. Based on our detection limit of 0.29 -fold coverage, the metagenomic approach described here could detect 1 ' $\mathrm{Ca}$. L. asiaticus' cell for every 52 phloem cells.

\section{DNA viruses and viroids not present.}

Because Koch's postulates have not been completed for HLB, it is possible that other agents, such as a virus or viroid, may be involved in the development of disease symptoms. To determine whether any such agent is present in HLB-infected phloem, all metagenomic sequence data was compared against a virus and viroid database. When a reference assembly of greening infected phloem sequences against the viroid database was performed, no sequencing reads assembled, indicating that there are no known viroids present within the phloem. This result is not unexpected because the phloem sample sequenced was of DNA extracted directly from the plant tissue and no reverse-transcriptase steps to obtain RNA sequences were performed. The reference assembly against the virus database did yield matches, but none showed good coverage. The highest fold coverage to any member of the virus database was 2.04-fold but the consensus sequence only spanned less than $1 \%$ of the reference that it matched (Supplementary Table S5). Considering the short nature of the consensus sequence and the fact that the match was to Hepatitis, a human virus, it is unlikely that this virus is present in the phloem sample. Although the amount of sequencing in this study may not be sufficient to detect low abundance viral particles, the amount of sequence obtained for this work should be sufficient to detect virus particles that are sufficiently abundant to cause disease. Therefore, DNA viruses are not likely to play a role in HLB in the phloem.

\section{High coverage of citrus.}

The DNA sequenced for this metagenomic analysis was extracted from total citrus phloem; therefore, it was expected that the dataset would include a high coverage of the $C$. sinensis genome in addition to the genomes of any microorganisms present. In order to confirm this, all phloem sequences were run through a reference assembly against a citrus expressed sequence tag (EST) database. Of the 34,505 EST in the database, $79.85 \%$ had hits from the phloem sequence, with an average fold coverage of 13.19 (results not shown).

\section{DISCUSSION}

Most of the research results that associate ' $\mathrm{Ca}$. Liberibacter spp.' to HLB diseases in citrus involve microscopic examinations, cloning, and sequencing of PCR-amplified DNA from infected tissue and other PCR-based techniques. In this study, a PCR independent metagenomic approach was taken to confirm the presence of ' $C a$. L. asiaticus' contigs, determine whether other organisms may be present, and assess the microbial diversity in HLB-infected phloem. Prior to confirming the role of ' $C a$. L. asiaticus' in infected citrus phloem, it was first necessary to verify that the contigs for this strain's genome in GenBank were only present in infected tissue. To that end, PCR performed with primers designed to each of the ' $\mathrm{Ca}$. L. asiaticus' contigs confirmed that 30 of the 32 contigs were present in HLB-infected but not healthy citrus tissue.
Once it was confirmed that the ' $\mathrm{Ca}$. L. asiaticus' contigs were present only in HLB-infected tissue, the presence of those contigs in infected citrus phloem was examined by metagenomics. In this analysis, all contigs were present in infected phloem with fold coverage ranging from 20.642 to 60.067. Such high fold coverage of these contigs from HLBinfected phloem further confirms the validity of these contigs belonging to the genome of ' $\mathrm{Ca}$. L. asiaticus' and a causative agent of HLB. It is unlikely that the high coverage of ' $\mathrm{Ca}$. L. asiaticus' is due to the presence of another alphaproteobacterium in the tissue because a selection of genomes from five different alphaproteobacterial relatives of ' $\mathrm{Ca}$. Liberibacter spp.' did not have greater than 0.2-fold coverage.

Because Koch's postulates have not been completed for ' $\mathrm{Ca}$. Liberibacter spp.' in HLB disease, it is possible that another pathogen or pathogens may play a role in disease development. For this reason, the composition of the microbial community in infected citrus phloem was also investigated by assembling the citrus phloem metagenome sequences against the 16S rRNA database from RDP. This analysis was run with data from three different high-throughput sequencing methods separately as well as with all the data combined and, in each case, the ' $\mathrm{Ca}$. L. asiaticus' was found to be the only bacterium with significant coverage of its $16 \mathrm{~S}$ rRNA gene. For all other bacteria in the RDP database, metagenomic sequences matched no greater than $13.5 \%$ of the $16 \mathrm{~S}$ gene or a fold coverage of 1.45 . If any other bacteria were present within the citrus phloem, a more even coverage over the entire 16S rRNA gene would be expected because the total DNA extract from infected phloem was sequenced without using any PCR amplification that would create biases. Therefore, it can be said with confidence that the matches of the contigs and 16S rRNA gene with the metagenomic dataset from HLB-infected phloem are indeed due to the presence of ' $C a$. L. asiaticus' in infected phloem and not another bacterium. In addition, no known DNA virus was identified in the metagenomic analysis although, in the field, most HLB-infected trees are also infected with CTV. However, the samples examined were chosen because they are free of CTV. We have examined the interaction of CTV and HLB and found little or no synergistic interactions (Folimonova et al. in press). Therefore, viruses were discounted as playing a role in HLB.

The results from this study differ slightly from others assessing the microbial diversity of HLB-infected citrus plants. In the current study, the only bacterium found to be present in infected citrus was ' $\mathrm{C}$. . L. asiaticus' whereas Sagaram and associates (2009) found a large diversity of microorganisms, comprising 47 orders of bacteria. However, two very different samples were analyzed in these studies. Sagaram and associates (2009) were looking at entire leaf midribs whereas the current study looked at phloem scraped from the inside of $C$. sinensis bark that was pealed from growing trees. The entire midrib includes the apoplast, which is known to harbor bacterial endophytes. It is likely that a larger array of bacteria are present in leaf tissue than are actually inside the phloem cells of the plant vascular system. The causative agent of HLB is thought to be restricted to phloem sieve tubes; therefore, this tissue is the ideal for examining HLB-associated DNA sequences. Because ' $C a$. Liberibacter spp.' are intracellular within phloem cells, being spread systemically from the site of infection to other tissues, there will be fewer bacteria present in this environment because any present would have to bypass more plant defense mechanisms to enter the cell. Although midribs do contain phloem tissue, other leaf tissue is also present in such preparations and would contain additional bacteria that would inflate the estimate of microbial diversity directly in contact with ' $\mathrm{Ca}$. L. asiaticus'. 
In addition, Chen and associates (2009) reported a strain of ' $C a$. P. asteri' associated with HLB-infected citrus that was also not identified in the current study. Because that study also examined leaf midribs as opposed to phloem cells from the bark, differences in the bacteria identified are not surprising. Although this study also reported observing phytoplasma in phloem sieve tube cells from midribs using electron microscopy, the majority of the microscopic examinations were performed in periwinkle, which showed an enrichment of the bacterium compared with citrus. Furthermore, the identification of phytoplasma in sieve tubes was done on the basis of cell wall thickness, without any molecular means of confirming their identity such as a phytoplasma-specific antibody. Thus, it is not certain that the phytoplasma are present in the sieve tube cells of citrus. However, these results do not preclude the possibility that phytoplasma can cause citrus-greening-like symptoms in citrus in some areas. In this work, citrus is shown to have these symptoms with ' $\mathrm{Ca}$. Liberibacter spp.' exclusively.

In another study, Davis and associates (2008) looked specifically at phloem tissue in an attempt to culture ' $\mathrm{Ca}$. L. asiaticus' and obtained a co-culture of ' $\mathrm{Ca}$ L. asiaticus' with actinobacteria related to $P$. acnes based on $16 \mathrm{~S}$ rRNA analysis, with a 1,006-bp 16S rRNA sequence from the co-culture having $100 \%$ identity to $P$. acnes. These observations are in contrast to the results of the present study because there was no significant coverage of the $P$. acnes genome in the metagenomic sequences. Although ' $\mathrm{Ca}$. L. asiaticus' was reported to have been co-cultured with $P$. acnes, it took 31 culturing attempts to obtain a second co-culture with these bacteria and 12 additional attempts to obtain a third co-culture from HLB-infected plants (Davis et al. 2008). The number of repeated attempts necessary to obtain co-cultures with $P$. acnes from HLB-infected tissue indicates that this bacterium may not be a common inhabitant of citrus. If $P$. acnes was commonly found in citrus plants and capable of supporting ' $\mathrm{C}$. . L. asiaticus' in co-culture, a higher abundance of $P$. acnes DNA would be expected in these plants. In addition, Davis and associates (2008) used $P$. acnes-specific primers to detect the bacterium in DNA extracts from citrus but only three healthy plants were reported to be positive for $P$. acnes. Also, whereas Davis and associates (2008) state that they identified $P$. acnes in their co-cultures, they did not specify whether PCR was performed to identify $P$. acnes in DNA directly isolated from HLB-infected plants. Furthermore, $P$. acnes is a commensal of human skin (Brüggemann et al. 2004), and its identification in plant tissues may be the result of contamination while handling the samples. In this work, $P$. acnes was not found in the metagenomic sequences and may not be associated with ' $\mathrm{Ca}$. L. asiaticus' in planta.

Given the large amount of sequencing data generated by the three high-throughput sequencing methods, there is a high likelihood that the genome of any bacteria present in the phloem sample would have been detected. To test this, a detection limit of three standard deviations above the mean observed fold coverage of a bacterial genome was used to distinguish between the false and genuine sequence matches to the ' $\mathrm{Ca}$. L. asiaticus' genome. Only the 'Ca. L. asiaticus' genome sequences met this standard. In addition, the level of $16 \mathrm{~S}$ rRNA coverage by other bacteria was also much lower than expected for a bacterium to be present in these samples. In both cases, when looking at entire genomes or specifically at 16S rRNA genes, ' $C a$. Liberibacter' was the only bacterium identified above these detection limits. Furthermore, fold coverage of the ' $\mathrm{Ca}$. Liberibacter' 16S rRNA and genome were 12 and 90 times higher than these limits, respectively. Because ' $\mathrm{Ca}$. L. asiaticus' occurs in very low concentrations in infected citrus (Tatineni et al. 2008) and was identified at such high levels above the detection limit, we are confident that any other low-abundance bacteria present in the tissue would have been detected by this method.

This article presents a new approach to evaluating the microbial diversity present in citrus tissue by a PCR-independent metagenomic method. This method has the advantage of avoiding biases based on PCR amplification and cloning (Polz and Cavanaugh 1998). In addition, by using high-throughput sequencing methods, a greater sequencing depth of the entire citrus phloem sample was obtained at a lower per-base cost than traditional Sanger sequencing. Similar metagenomics studies have also been applied to investigate the causative agents of other diseases. Using 454 pyrosequencing, colony collapse disorder (CCD) in honey bees (Apic mellifera) was found to be associated with Israeli acute paralysis virus (IAPV), an unclassified dicistrovirus (Cox-Foster et al. 2007). In that study, results from the metagenomic analysis contrasted with previously proposed causative agents of CCD. Although IAPV sequences were found only in CCD cases, Nosema ceranae and an Iflavirus sp. proposed to be associated with the disease were identified in both CDD and non-CCD cases (Cox-Foster et al. 2007). As a result, this demonstrates how the metagenomics approach can be used to pinpoint disease agents free from culturing, PCR, and cloning biases.

In summary, this study used a culture- and PCR-independent metagenomic approach to verify the validity in citrus of the reported ' $\mathrm{Ca}$. L. asiaticus' contigs that were obtained from infected psyllids, determine that it is the major and, perhaps, the only bacterium present in the phloem of infected citrus phloem, and thereby substantiate its proposed role as the likely causative agent of HLB. With the decreasing costs in sequencing due to high-throughput methods and the level of sequence coverage of samples analyzed, metagenomics will become a most effective method for studying uncultured plant pathogens in the future. This will allow researchers to examine a broader view of the complexity of organisms present in the diseased state.

\section{MATERIALS AND METHODS}

\section{Sample preparation and DNA extraction.}

Eleven citrus plants, including C. volkameriana, C. aurantifolia cv. Swingle, C. limon cv. Eureka, 'Hirado Buntan Pink,' and $C$. sinensis cv. Valencia, symptomatic for citrus greening, were sampled. Branches were cut from plants and surface sterilized by wiping with tissue paper and $70 \%$ ethanol. The bark, which was 'slipping' in growing plants, was removed and the phloem cells were scraped off by using a sterile, disposable scalpel. DNA was extracted directly from the phloem cells.

DNA was isolated from the collected phloem tissue samples (approximately $150 \mathrm{mg}$ ) using the FastDNA Kit (Qbiogene, Inc., Carlsbad, CA, U.S.A.). After the DNA extraction, samples were purified with the DNeasy Tissue kit (Qiagen, Valencia, CA, U.S.A.) following the manufacturer's instructions. This procedure was repeated to obtain enough DNA for 454, SOLiD, and Solexa sequencing.

\section{PCR to confirm validity}

of ' $\boldsymbol{C a}$. L. asiaticus' contigs in GenBank.

DNA extracted from healthy and infected $C$. sinensis cv. Valencia (as described above) was used as template in PCR reactions with primer pairs for each ' $\mathrm{Ca}$. L. asiaticus' contig in GenBank. Primers were designed to amplify a $0.5-, 1-$, or $2-\mathrm{kb}$ fragment from each contig using Primer3 (v. 0.4.0) (Supplementary Table S6). PCR reactions contained $4 \mu$ of $5 \times$ HF Phusion Buffer (New England Bioloabs, Ipswich, MA, U.S.A.), $200 \mu \mathrm{M}$ each dNTP, $2.5 \mu \mathrm{M}$ each primer, $0.4 \mathrm{U}$ of Phusion polymerase, and approximately $50 \mathrm{ng}$ of DNA template in a final volume of $20 \mu \mathrm{l}$. PCR reactions were held at 
$98^{\circ} \mathrm{C} 30 \mathrm{~s}$; followed by 30 cycles of $98^{\circ} \mathrm{C}$ for $10 \mathrm{~s}, 60^{\circ} \mathrm{C}$ for 30 $\mathrm{s}$, and $72^{\circ} \mathrm{C}$ for $1 \mathrm{~min}$; with a final extension at $72^{\circ} \mathrm{C}$ for 10 min. PCR products from healthy and HLB-infected samples were resolved on a $1 \%$ agarose gel and examined for bands in the expected size range.

\section{ARISA analysis, cloning, and sequencing for initial assessment of bacterial diversity and to confirm presence of ' $\mathrm{Ca}$. $\mathrm{L}$. asiaticus'.}

Bacterial community composition was assessed by ARISA (Fisher and Triplett 1999). ARISA is a molecular technique for constructing bacterial community fingerprints based on the length heterogeneity of the intergenic transcribed spacer region of bacterial rRNA operons (Bosshard et al. 2000). Treating the elements of ARISA profiles as operational taxonomic units allows for bacterial community comparisons. In this study, ARISA profiles were assumed to be indicative of bacterial community composition, and differences in ARISA profiles were assumed to reflect variation in the composition of the respective bacterial communities.

PCR reaction mixtures contained $5 \mu \mathrm{l}$ of $10 \times$ PCR buffer (Promega, Madison, WI, U.S.A.), $200 \mu \mathrm{M}$ each dNTP, $100 \mu \mathrm{M}$ each primer, $2.5 \mathrm{U}$ of Taq polymerase, and approximately 100 ng of DNA template (estimated by using NanoDrop ND-1000 spectrophotometer) in a final volume of $50 \mu \mathrm{l}$. The primers used were 787F (5'-ATTAGATACCCNGGTAG-3') (Roesch et al. 2007) and L-D-Bact-132-a-A-18 (5'-CCGGGTTTCCCCA TTCGG-3') (Ranjard et al. 2001). Reaction mixtures were held at $94^{\circ} \mathrm{C}$ for $3 \mathrm{~min}$; followed by 30 cycles of amplification at $94^{\circ} \mathrm{C}$ for $45 \mathrm{~s}, 55^{\circ} \mathrm{C}$ for $1 \mathrm{~min}$, and $72^{\circ} \mathrm{C}$ for $2 \mathrm{~min}$; and a final extension of $72^{\circ} \mathrm{C}$ for $7 \mathrm{~min}$.

Sample fragments were then discriminated by using on-chip gel electrophoresis with the Agilent 2100 Bioanalyzer and DNA LabChip kit 7500. Briefly, a ladder with known fragment sizes is loaded and a standard curve of migration time versus fragments size is plotted. The size of each fragment in the sample is then calculated by taking into account the migration times measured. Lower and upper marker standards are also run with each sample in order to align the ladder data with data from the sample wells. Data is translated into gel-like images (bands) and electrophelograms (peaks).

After the dominant peaks were detected, the PCR products were loaded in a $1.2 \%$ agarose gel and the bands were identified and excised. The bands were purified using QIAEX II Gel Extraction Kit (Qiagen) following the manufacturer's instructions. The purified fragments were cloned into a TOPO TA Cloning (Invitrogen, Carlsbad, CA, U.S.A.). Plasmids were purified with the QIAprep spin miniprep kit (Qiagen) and gene fragments were cycle sequenced using T7 primer in a DYEnamic ET terminator cycle sequencing kit (GE Healthcare, Piscataway, NJ, U.S.A.) on a PTC200 thermocycler (Bio-Rad, Hercules, CA, U.S.A.) and run on a 96-well MegaBACE 1000 capillary sequencer (GE Healthcare). Once the presence of 'Ca. Liberibacter spp.' was confirmed by sequencing, DNA from one positive tree was used for further analysis.

\section{ARISA sequence analysis and classification.}

The sequences obtained for each excised band were initially analyzed by using VecScreen, an on-line tool for identifying segments of a nucleic acid sequence that may be of vector origin. After manual edition for the elimination of potentially chimeric sequences, phylogenetic analyzes were conducted using MEGA version 4 (Tamura et al. 2007). The evolutionary distance among the sequences was inferred using the neighborjoining method (Saitou and Nei 1987), and a bootstrap test (500 replicates) was conducted in order to calculate the confidence limits of the phylogeny (Felsenstein 1985). All positions containing gaps and missing data were eliminated from the dataset (complete deletion option).

\section{High-throughput sequencing.}

Three high-throughput sequencing methods were used to assess the bacterial diversity of a symptomatic $C$. sinensis cv. Valencia plant. DNA from one ' $\mathrm{Ca}$. Liberibacter sp.'-positive plant was used for 454 pyrosequencing, Solexa, and SOLiD sequencing. Analyses were performed with DNA extracted from an infected citrus tree positive for citrus greening according to the ARISA analysis, cloning, and sequencing of the DNA fragments. For $454,10 \mu \mathrm{g}$ of DNA was sent to the Interdisciplinary Center for Biotechnology Research (ICBR) at the University of Florida, prepared according to the manufacturer's instructions, and sequenced using the 454 Genome Sequencer FLX System (454 Life Sciences, Branford, CT, U.S.A.). An additional $10 \mu \mathrm{g}$ of DNA was sent to ICBR at the University of Florida and used for SOLiD sequencing according to the manufacturer's protocols (Applied Biosystems, Carlsbad, CA, U.S.A.). For Solexa sequencing, $10 \mu \mathrm{g}$ of DNA was sent to the Center for Genome Research and Biocomputing at Oregon State University, where it was prepared using the manufacturer's standard protocols and run on the Illumina $1 \mathrm{G}$ Genome Analyzer (Illumina, Hayward, CA, U.S.A.).

\section{Sequence analysis.}

Reads obtained from each sequencing method were analyzed using CLC Genomics Workbench Version 3.2. Parameters used were $99 \%$ similarity for 454 reads, a limit of 1 for Solexa and SOLiD, and a mismatch cost of 3. All other parameters were set to the program's default. Analyses of the data from each sequencing method were run individually and pooled together. To determine microbial community composition within C. sinensis phloem, all sequence data was first run through a reference assembly against the $C$. sinensis chloroplast genome from the National Center for Biotechnolgoy Information (NCBI) (NC_008334) to eliminate false positives from chloroplast rRNA. Sequencing reads that did not assemble to the chloroplast genome were used in a reference assembly against the $16 \mathrm{~S}$ database from the RDP. The reference assemblies were done on the entire dataset as well as individually for 454, Solexa, and SOLiD data. To determine whether any viruses or viroids were present in the phloem of the greening-infected plant, all sequencing reads were run in reference assemblies against viroid and virus databases. The viroid database was composed of all 39 completed viroid genomes from NCBI and the virus database was composed of all annotated virus genomes from the Viral Bioinformatics Resource Center.

To estimate the level of ' $\mathrm{Ca}$. L. asiaticus' genome coverage present in the phloem sequences, a reference assembly of the data was run against all ' $\mathrm{Ca}$ L. asiaticus' str. psy62 contigs available in GenBank (NZ_ABQW00000000). Four reference assemblies were performed with the putative ' $\mathrm{Ca}$. L. asiaticus' contigs, one each for 454, SOLiD, and Solexa data, as well as a collective reference assembly with all data from each method. Prior to performing the reference assemblies, the 34 ' $C a$. L. asiaticus' contigs from GenBank were assembled in SeqMan Pro Version 7.2.1 (DNAstar, Lasergene, Madison, WI, U.S.A.), which reduced the number of contigs to 32 .

In order to confirm that the ' $\mathrm{Ca}$. L. asiaticus' matches seen in citrus phloem were not the result of other alphaproteobacteria present in citrus phloem, reference assemblies with the sequenced genomes of five alphaproteobacterial relatives were performed. The genomes used in this analysis were selected by performing a local BLAST search of the ' $\mathrm{Ca}$ L. asiaticus' contigs against a database of 40 fully sequenced alphaproteobacteria, including a representative from each fully sequenced 
genus on NCBI (NC_009484.1, NC_003062.2, NC_004842.2, NC_009937.1, NC_008783.1, NC_010581.1, NC_004463.1, NC_010742.1, NC_002696.2, NC_009952.1, NC_007354.1, NC_007722.1, NC_011365.1, NC_006677.1, NC_008343.1, NC_008358.1, NC_007802.1, NC_007626.1, NC_008347.1, NC_002678.2, NC_010172.1，NC_007798.1，NC_007964.1, NC_007794.1, NC_009668, NC_009488.1, NC_008686.1, NC_009719.1，NC_007205.1，NC_011144.1，NC_008380.1, NC_007494.1, NC_008435.1，NC_007643.1，NC_009881.1, NC_008209.1, NC_009511.1, NC_002978.6, NC_009720.1, and NC_006526.1). The five species that were most closely related to ' $C a$. Liberibacter' were chosen for this study. Those five genomes were $R$. leguminosarum bv. viciae 3841 (NC_008378, NC_008379, NC_008380, NC_008381, NC_008382, NC_008383, and NC_008384), Bartonella bacilliformis KC583 (NC_008783.1), Caulobacter crescentus CB15 (NC_002696.2), Brucella abortus S19 (NC_010740 and NC_010742), and Orientia tsutsugamushi str. Boryong (NC_009488.1). 'Ca. P. asteri' and P. acnes have also been reported to be associated with HLB infection in citrus; therefore, reference assemblies were performed with fully sequenced strains of these species, ' $C a$. P. asteris' AYWB (NC_007716, NC_007717, NC_007718, NC_007719, and NC_007720) and $P$. acnes KPA171202 (NC_006085). An additional reference assembly was performed against the genome of the non-HLBassociated bacterium, M. luteus NCTC 2665 (NC_012803), as a comparison.

To determine the detection limit for identifying a bacterial genome present in the metagenomic data, the mean and standard deviations for the fold coverage of R. leguminosarum bv. viciae 3841, Bartonella bacilliformis KC583, C. crescentus CB15, Brucella abortus S19, O. tsutsugamushi str. Boryong, 'Ca. P. asteris' AYWB, and P. acnes KPA171202 were calculated. Because $99 \%$ percent of observed genomes are likely to fall within 3 standard deviations of the mean assuming a normal distribution, any genome with enough matching reads and bases to have a fold coverage greater than 3 standard deviations above the mean was considered significant. The detection limit for identifying a true match from the RDP database was determined using the same method by calculating the mean and standard deviation of the fold coverage from each match in the database.

With such a large amount of sequence data from citrus phloem, it is expected that the dataset also contains a high fold coverage of the citrus genome. To confirm this, a reference assembly of all citrus phloem data was performed against a citrus EST database. The citrus EST database was composed of all Citrus sinensis cv. Valencia EST from KEGG. These EST were run in a de novo assembly on CLC Genomics Workbench to eliminate duplicates. Assembled contigs and nonassembled EST were used as the reference in the assembly with the complete citrus phloem metagenome dataset.

\section{ACKNOWLEDGMENTS}

This research was supported by grants from the Florida Citrus Production Research Advisory Council, the Florida Department of Citrus (00079687), the Florida Agricultural Experiment Station, the National Science Foundation (MCB-0454030), and the United States Department of Agriculture (2005-35319-16300).

\section{LITERATURE CITED}

Bosshard, P. P., Stettler, R., and Bachofen, R. 2000. Seasonal and spatial community dynamics in the meromictic Lake Cadagno. Arch. Microbiol. 174:168-174.

Bove, J. M. 2006. Huanglongbing: A destructive, newly-emerging, centuryold disease of citrus. J. Plant Pathol. 88:7-37.
Bruggemann, H., Henne, A., Hoster, F., Liesegang, H., Wiezer, A., Strittmatter, A., Hujer, S., Durre, P., and Gottschalk, G. 2004. The complete genome sequence of Propionibacterium acnes, a commensal of human skin. Science 305:671-673.

Capoor, S. P., Rao, D. G., and Viswanat, S. M. 1967. Diaphorina citri Kuway., a vector of greening disease of citrus in India. Indian J. Agric Sci. 37:572-576.

Chen, J., Pu, X., Deng, X., Liu, S., Li, H., and Civerolo, E. 2009. A phytoplasma related to 'Candidatus Phytoplasma asteri' detected in citrus showing Huanglongbing (Yellow Shoot Disease) symptoms in Guangdong, P. R. China. Phytopathology 99:236-242.

Cox-Foster, D. L., Conlan, S., Holmes, E. C., Palacios, G., Evans, J. D., Moran, N. A., Quan, P. L., Briese, T., Hornig, M., Geiser, D. M., Martinson, V., vanEngelsdorp, D., Kalkstein, A. L., Drysdale, A., Hui, J., Zhai, J. H., Cui, L. W., Hutchison, S. K., Simons, J. F., Egholm, M., Pettis, J. S., and Lipkin, W. I. 2007. A metagenomic survey of microbes in honey bee colony collapse disorder. Science 318:283-287.

Davis, M. J., Mondal, S. N., Chen, H. Q., Rogers, M. E., and Brlansky, R. H. 2008. Co-cultivation of 'Candidatus Liberibacter asiaticus' with Actinobacteria from Citrus with Huanglongbing. Plant Dis. 92:1547-1550.

Doddapaneni, H., Liao, H., Lin, H., Bai, X., Zhao, X., Civerolo, E. L., Irey, M., Coletta-Filho, H., and Pietersen, G. 2008. Comparative phylogenomics and multi-gene cluster analyses of the Citrus Huanglongbing (HLB)-associated bacterium Candidatus Liberibacter. BMC Res. Notes 1:72.

Duan, Y., Zhou, L., Hall, D. G., Li, W., Doddapaneni, H., Lin, H., Liu, L., Vahling, M., Gabriel, D. W., Williams, K. P., Dickerman, A., Sun, Y., and Gottwald, T. Complete genome sequence of citrus Huanglongbing bacteirum, 'Candidatus Liberibacter asiaticus' obtained thorugh metagenomics. Mol. Plant-Microbe Interact. 22:1011-1020.

Felsenstein, J. 1985. Confidence limits on phylogenies: An approach using the bootstrap. Evolution 39:783-791.

Fisher, M. M., and Triplett, E. W. 1999. Automated approach for ribosomal intergenic spacer analysis of microbial diversity and its application to freshwater bacterial communities. Appl. Environ. Microbiol. 65:46304636.

Folimonova, S. Y., Robertson, C. J., Garnsey, S. M., Gowda, S., and Dawson. W. O. 2009. Examination of the responses of different genotypes of citrus to Huanglongbing (citrus greening) under different conditions. Phytopathology. In press.

Garnier, M., and Bove, J. M. 1983. Transmission of the organism associated with citrus greening disease from sweet orange to periwinkle by dodder. Phytopathology 73:1358-1363.

Garnier, M., Danel, N., and Bove, J. M. 1984. Aetiology of citrus greening disease. Ann. Microbiol. 135A:169-179.

Ghosh, S. K., Giannotti, J., and Louis, C. 1978. Multiplication intensive des prokaryotes associés aux maladies de type "greening" des agrumes dans les cellules criblées de cuscute. Ann. Phytopathol. 9:525-530.

Jagoueix, S., Bove, J. M., and Garnier, M. 1994. The phloem-limited bacterium of greening disease of citrus is a member of the $\alpha$ subdivision of the Proteobacteria. Int. J. Syst. Bacteriol. 44:379-386.

Jagoueix, S., Bove, J. M., and Garnier, M. 1997. Comparison of the $16 \mathrm{~S} / 23 \mathrm{~S}$ ribosomal intergenic regions of "Candidatus Liberobacter asiaticum" and "Candidatus Liberobacter africanum," the two species associated with citrus Huanglongbing (greening) disease. Int. J. Syst. Bacteriol. 47:224-227.

Lin, H., Doddapaneni, H., Bai, X. J., Yao, J. Q., Zhao, X. L., and Civerolo, E. L. 2008. Acquisition of uncharacterized sequences from Candidatus Liberibacter, an unculturable bacterium, using an improved genomic walking method. Mol. Cell Probes 22:30-37.

McClean, A. P. D., and Oberholzer, P. C. J. 1965. Citrus psylla, a vector of the greening disease of sweet orange. S. Afr. J. Agric. Sci. 8:298-298.

Polz, M. F., and Cavanaugh, C. M. 1998. Bias in template-to-product ratios in multitemplate PCR. Appl. Environ. Microbiol. 64:3724-3730.

Ranjard, L., Poly, F., Lata, J. C., Mougel, C., Thioulouse, J., and Nazaret, S. 2001. Characterization of bacterial and fungal soil communities by automated ribosomal intergenic spacer analysis fingerprints: Biological and methodological variability. Appl. Environ. Microbiol. 67:4479-4487.

Roesch, L. F., Fulthorpe, R. R., Riva, A., Casella, G., Hadwin, A. K. M., Kent, A. D., Daroub, S. H., Camargo, F. A. O., Farmerie, W. G., and Triplett, E. W. 2007. Pyrosequencing enumerates and contrasts soil microbial diversity. ISME J. 1:283-290.

Sagaram, U. S., DeAngelis, K. M., Trivedi, P., Andersen, G. L., Lu, S. E., and Wang, N. 2009. Bacterial diversity analysis of Huanglongbing pathogen-infected citrus, using PhyloChip arrays and 16S rRNA gene clone library sequencing. Appl. Environ. Microbiol. 75:1566-1574.

Saitou, N., and Nei, M. 1987. The neighbor-joining method: A new method for reconstructing phylogenetic trees. Mol. Biol. Evol. 4:406-425.

Tamura, K., J. Dudley, J., Nei, M., and Kumar, S. 2007. MEGA4: Molecular evolutionary genetics analysis (MEGA) software version 4.0. 
Mol. Biol. Evol. 24:1596-1599.

Tatineni, S., Sagaram, U.S., Gowda, S., Robertson, C. J., Dawson, W. O., Iwanami, T., and Wang, N. 2008. In planta distribution of 'Candidatus Liberibacter asiaticus' as revealed by polymerase chain reaction (PCR) and real-time PCR. Phytopathology 98:592-599.

Teixeira, D. D., Saillard, C., Eveillard, S., Danet, J. L., da Costa, P. I., Ayres, A. J., and Bove, J. 2005. 'Candidatus Liberibacter americanus', associated with citrus huanglongbing (greening disease) in Sao Paulo State, Brazil. Int. J. Syst. Evol. Microbiol. 55:1857-1862.

Teixeira, D. C., Wulff, E. C., Martins, E. W., Kitajima R., Bassanezi, R., Ayres, A. J., Eveillard, S., Saillard, C., and Bové, J. M. 2008. A phytoplasma closely related to pigeon pea witches'-broom phytoplasma (16Sr IX) is associated with citrus huanglongbing symptoms in the state of São Paulo, Brazil. Phytopathology 98:977-984.
AUTHOR-RECOMMENDED INTERNET RESOURCES

CLC Genomics Workbench: www.clcbio.com

Kyoto Encyclopedia of Genes and Genomes (KEGG) Citrus sinensis webpage: www.genome.jp/kegg-bin/show_organism?org=ecsi

National Center for Biotechnology Information VecScreen database: www.ncbi.nlm.nih.gov/VecScreen/VecScreen.html

Oregon State University Center for Genome Research \& Biocomputing website: www.cgrb.oregonstate.edu

Primer3 database: frodo.wi.mit.edu

The Ribosomal Database Project (RDP): rdp.cme.msu.edu

University of Florida Interdisciplinary Center for Biotechnology Research website: www.biotech.ufl.edu

Viral Bioinformatics Resource Center: athena.bioc.uvic.ca 